\title{
Monitoring Servers, With a Little Help from my Bots
}

\author{
Takashi Yamanoue \\ Fukuyama University \\ Sanzo, 1, Gakuen-cho \\ Fukuyama, Hiroshima, 729-0292 \\ yamanoue@fukuyama-u.ac.jp
}

\begin{abstract}
This paper discusses a method of employing bots instead of people to monitor servers or server rooms. A bot is a remote controlled computer or a remote controlled program. A bot is usually a malicious program which is an element of a botnet. A botnet is used for doing malicious things such as spreading spam mails or doing DDoS Attacks. We have made bots and we are using bots for doing beneficial things such as monitoring a server instead of doing malicious things. We are monitoring a web server in our campus using a bot. This bot is tweeting whether the server is running or not periodically on the twitter. We are also monitoring a server room in our campus using another bot. This bot shows managers transition of the room temperature and others.
\end{abstract}

\section{CCS CONCEPTS}

- Social and professional topics $\rightarrow$ Professional topics $\rightarrow$ Management of computing and information systems $\rightarrow$ System management $\rightarrow$ Centralization / decentralization, Network operations

\section{KEYWORDS}

Bot, Monitoring, Management, Twitter, Wiki, Raspberry Pi.g

\section{INTRODUCTION}

Information and Communication Technology infrastructure managers of universities are always monitoring many servers and server rooms in their campus[2][3]. Monitoring a server or a server room seems easy work for people who have never worked at an ICT administration department. However, we know, it is hard work. If we failed to identify potential trouble with a server or server room, or if we could not cope with the trouble, users can't do their business. It could also cause a serious trouble such as a fire in a building.

\footnotetext{
Permission to make digital or hard copies of all or part of this work for personal or classroom use is granted without fee provided that copies are not made or distributed for profit or commercial advantage and that copies bear this notice and the full citation on the first page. Copyrights for components of this work owned by others than ACM must be honored. Abstracting with credit is permitted. To copy otherwise, or republish, to post on servers or to redistribute to lists, requires prior specific permission and/or a fee. Request permissions from Permissions@acm.org.

SIGUCCS 2017, October 1-4, 2017, Seattle, WA, USA

(C) 2017 Association for Computing Machinery.

ACM ISBN 978-1-4503-4919-2/17/10 . . \$15.00

https://doi.org/DOI: 10.1145/3123458.3123461
}

No one is perfect, so an ICT infrastructure manager could potentially fail to identify trouble with a server or the server room. So managers are using various ways to identify trouble in advance.

As a way of monitoring servers or server rooms, we show a way of employing bots instead of employing people to monitor servers or server rooms. A bot is a remote-controlled computer or a remote-controlled program. A bot is usually a malicious program which is an element of a botnet[1]. We use the bot for beneficial things[4][5][6][7].

We are monitoring a Web server in our campus using a bot. This bot is tweeting whether the server is running or not periodically on Twitter. We are also monitoring a server room in our campus using another bot. This bot shows managers changes in the room temperature and the room brightness. These bots are doing a good job.

Our monitoring way is an Internet of Things (IoT) because bots of the way are not operated by a person directly and they are connected to the Internet.

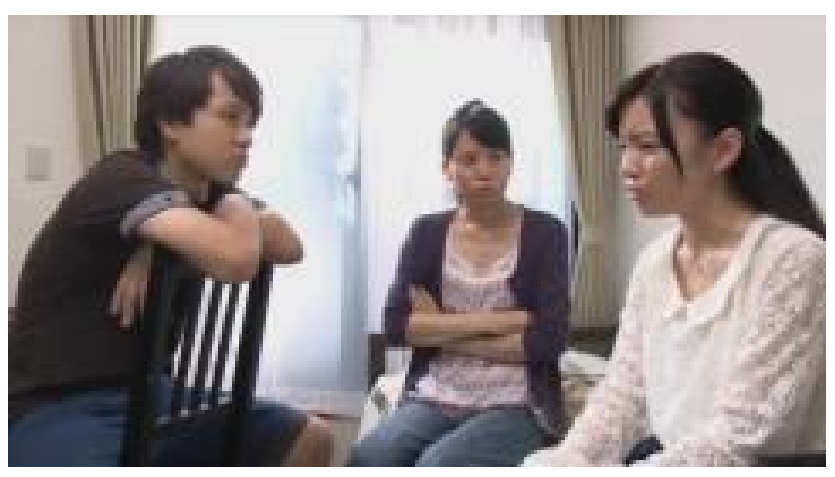

Figure 1: A scene of the computer ethics video clips.

\section{TROUBLES WITHOUT MY BOTS}

\section{$2.1 \quad$ A Server Trouble}

We are using computer ethics video clips for enhancing cyber security of our campus (Figure 1)[8][9]. The video clips were installed in a Web server of our campus and we can't access the Web server from the outside of our campus because of the license of the video clips.

The video clips are used in the classes of the author and other faculties in out campus. The Web server is maintained by the author. 
When the author went to overseas business trip, the author received a mail from a faculty member of our university, which told the author that the Web server was down and asked the author to cope with the situation.

At that time, the author forgot to confirm that the Web server is working or not. Even if the author remembers that the author should confirm that the server is working or not, every day, the author could not confirm the Web server because the author was not in the campus.

After all, the author asked his students to cope with the situation.

\subsection{A Server Room Trouble}

There was air conditioner problem in a server room in our campus. The air conditioner malfunctioned during the weekend and no one could notice this trouble until a Monday class, which uses PCs in a computer laboratory. Servers in the server room also malfunctioned because of the high temperature in the room. There could be a fire in the room if there had been a longer delay in noticing the problem.

As a result of the server malfunctions, we could not use the computer laboratories at that time, because PCs use the Active Directory servers and file servers in the server room.

A server room usually has no window and no one in the server room except when someone maintains servers. So, it is hard to know server room conditions from the outside without using appropriate tools.

There are commercial services for monitoring a server from outside. These services usually require installing a monitoring program in the server. However, it is hard to install such programs in a server which is not a property of our university.

We have called the maintenance company of the air conditioner and another maintenance company of the servers at that time. Fortunately, they fixed the trouble in a few days.

\section{WHAT ARE MY BOTS}

We are using bots for doing beneficial things such as to cope with such troubles in the section two instead of doing malicious things. Some bots are Raspberry PIs with the bot software. Others are android smart phones with sensors and the bot software. These bots are controlled by commands and programs in Wiki pages of web servers. Managers can control bots in a LAN, which is protected from the outside by NAT routers, from the outside of the LAN, by writing commands and programs in Wiki pages of web servers which is located at the outside of the LAN or which can be accessed from the outside of the LAN.

When a server was connected to a campus LAN, which cannot be accessed from the Internet directly, it is impossible to monitor the server from the outside of the campus directly. However, the ability to monitor the server from outside of campus would be convenient for times the manager is not on campus. A bot can help the manager to monitor the server in such cases. A server room is usually isolated from outside and there is no one in the server room. A bot also can help the manager to monitor the server room. There may be a server which is maintained by a third party company and the manager cannot install monitoring software into the server. A bot can help the manager to monitor the server in such cases.

Figure. 2 shows the behavior of our bot. The bot repeats the following.

1) Wait for a moment.

2) Read commands and a program from the Wiki page from the specific Wiki site which is assigned to the bot.

3) Execute these commands and the program. The program can read other Wiki pages and Web pages. The program can read tweets from Twitter and can tweet on Twitter. If the bot has sensors, it also can read data from the sensors. If the bot has actuators, it can send data to the actuators.

4) Write results of the execution to the send buffer. Data in the send buffer are written back to the Wiki page which contains the commands and the program.

The series of commands on the wiki page is read and interpreted when the page has it. If a program is embedded in the series of commands, then the program is transferred to the language processor of the bot. The program is translated into its internal representation. The internal representation of the program is evaluated by the interpreter.

Figure 3 shows an example of a program which is embedded in a series of commands. In this example, lines which start with "command:" are the commands. The first line,

command: set readInterval $=3600000$

shows that the mobile terminal reads the page of the given URL every one hour after that. The right hand side of the equation shows the interval time in milliseconds. Lines which start with "program:" are lines of the program. A program is enclosed by the following two command lines. In this example, the program is named "ex" by these lines.

command: program ex

command: end ex

The last command line,

command: run ex

shows that the program "ex" is translated into its internal representation and executed after that when this command line is interpreted. Figure 4 shows the output of the program.

A Wiki page for the bot also can contain the "set pageName" command and the "include" command. When the "set pageName" command is interpreted in the bot, the bot will use the Wiki page which is designated by the set pageName command, as the main Wiki page, next time. The page name of the designated Wiki page can include the current time or the current date. For example, when the following command was interpreted at the time of eight o'clock, the bot use the page of "pir-1-8", in the same Wiki server of the current Wiki page, as the main Wiki page, next time. 


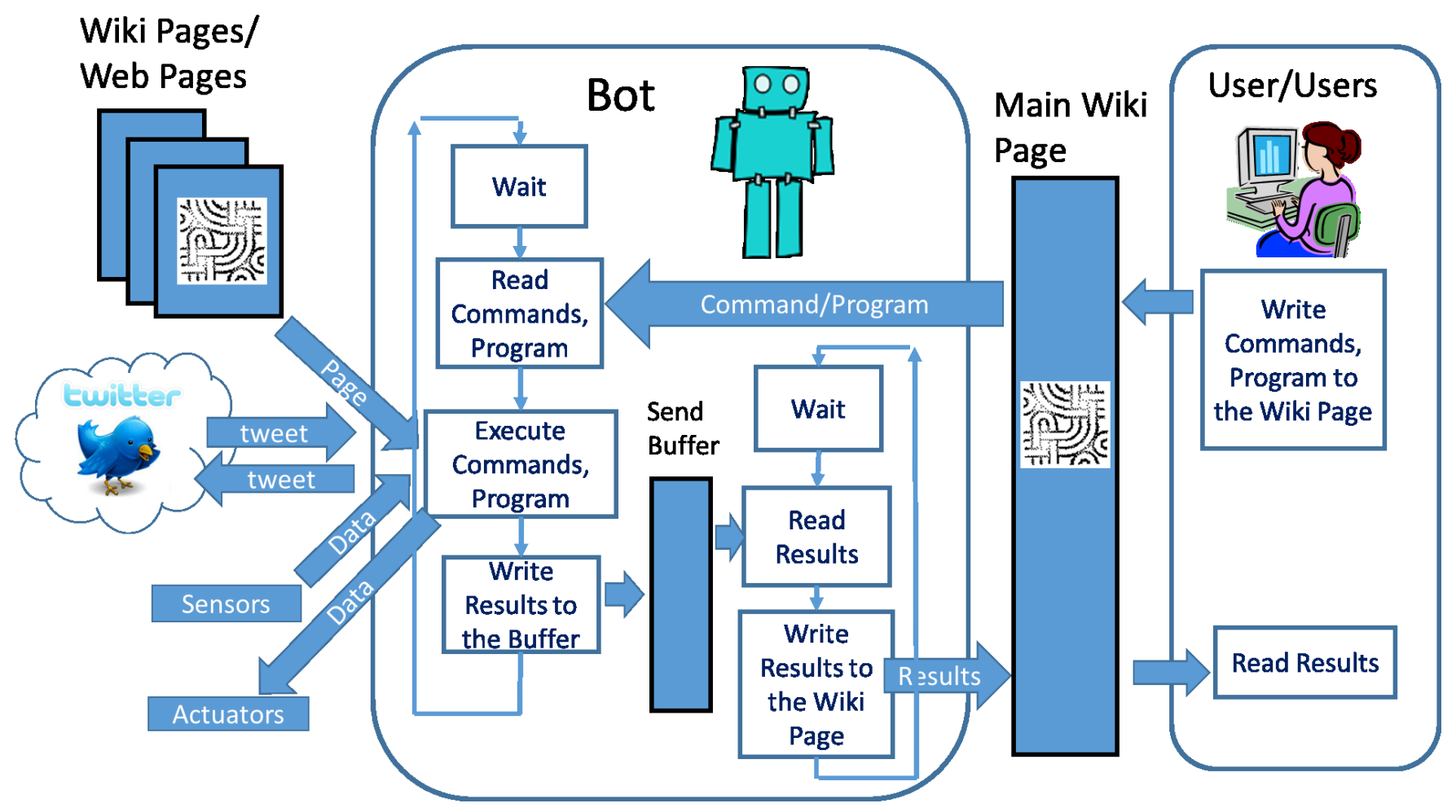

Figure 2: Behavior of a bot.

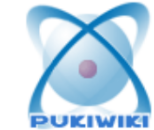

\section{wiki-basic-ex1}

[ Front page ] [ Edit | Freeze | Diff | Backup | Upload | Reload ] Help ]

\begin{tabular}{|l|l|l|l|}
\hline index & $\begin{array}{l}\text { command: set readinterval=3600000 } \\
\text { command: program ex } \\
\text { program: sum=0 } \\
\text { program: for } i=1 \text { to } 10 \\
\text { program: sum=sum+i } \\
\text { prontPage }\end{array}$ \\
\hline simple ex & $\begin{array}{l}\text { program: next } i \\
\text { command: end ex } \\
\text { ample }\end{array}$ \\
\hline music & \\
\hline
\end{tabular}

Figure 3: Example of a program which is embedded in a series of commands.

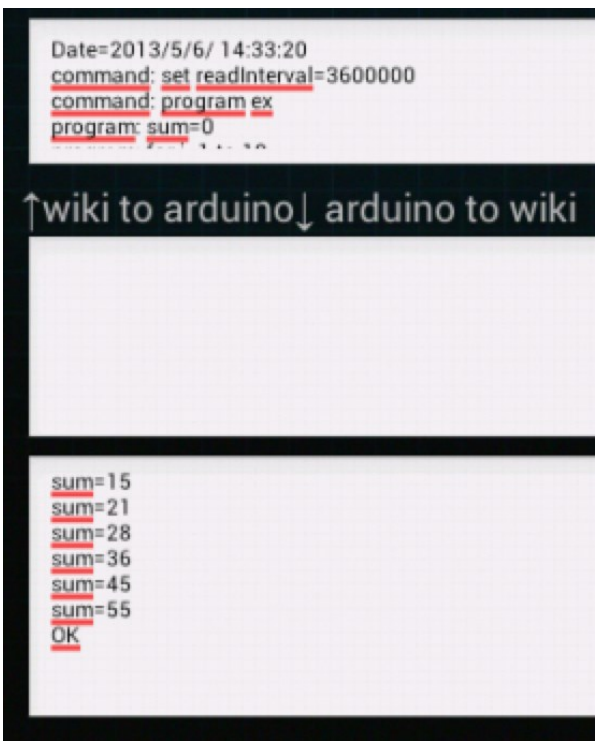

Figure 4: The output of the program of Figure 6. 


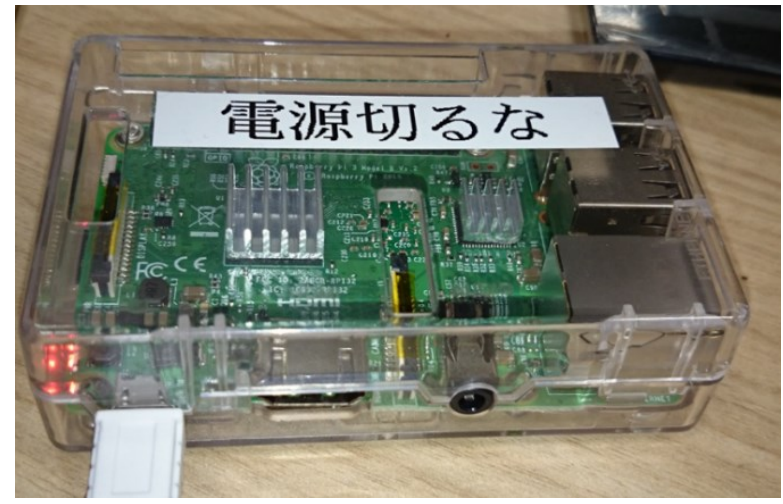

Figure 5: RPi-Bot, Raspberry Pi with the bot program.

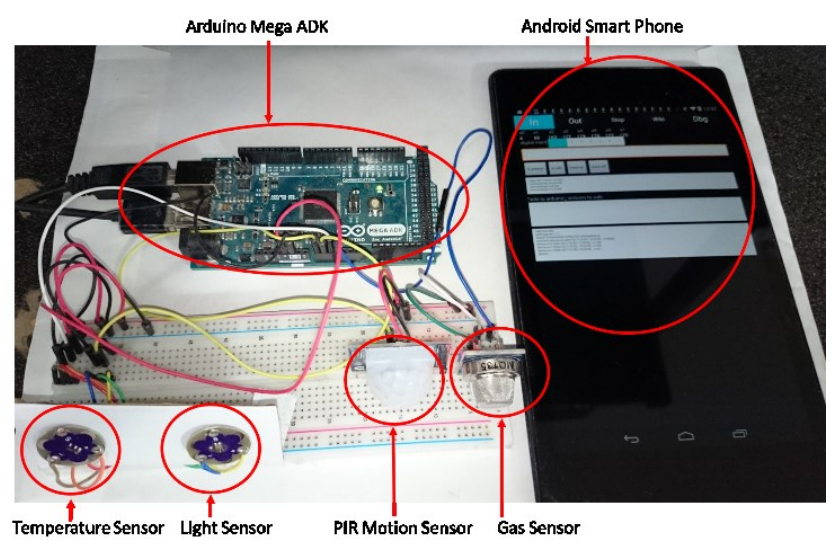

Figure 6: ADK-Bot, Android smartphone with the bot program which is equipped with an Arduino board with sensors.

set pageName="pir-1-<hour $>"$

When the "include" command is interpreted in the bot, the bot insert the Wiki page, which is designated by the include command, into the place at the include command of the original Wiki page. This command is useful when there are identical commands or program in many of Wiki pages.

We call the Wiki page, which contains the commands, the program, and data which are write backed from the bot, as the main Wiki page. Commands and the program of the main Wiki page can be modified to change the behavior of the bot without stopping the bot.

The bot can be connected to a LAN which is protected by a router with the NAT or NAPT function. The manager of the bot can control the bot from the outside of the LAN.

We have developed two kinds of bots. One kind of them is $R P i-B o t$, a Raspberry Pi with the bot program for the Raspberry $\mathrm{Pi}$ (Figure 5), and another kind of them is $A D K-B o t$, an android smartphone with the bot program for the android, which is equipped with an Arduino board with sensors (Figure 6). ADKBot has a temperature sensor, a light sensor, a PIR motion sensor and a Gas sensor. The PIR motion sensor detects whether a person is near the sensor or not. The Gas sensor detects the air pollution level.

\section{EMPLOYMENT OF MY BOTS}

We have made two kinds of monitoring system by employing two kinds of bots. One kind of the monitoring system is the Web server monitoring system which monitors whether the Web server is running or not. Another kind of the monitoring system is the server room monitoring system which monitors the transition of temperature and other environmental factors in a server room.

\subsection{Web Server Monitoring System}

The Web server monitoring system consists of a RPi-Bot, A Wiki page of PukiWiki software and Twitter (Figure 7). The system monitors our Web server which contains the computer ethics video clips. The bot is located in the campus LAN in order to see the status of the Web server which can not seen from the outside of the LAN. The bot repeats to read the Wiki page which contains the program for monitoring the Web server, and to execute the program. The bot monitors whether the Web server is running or not, and reports the status of the Web server to managers by tweeting on Twitter directed by the program.

Figure 8 shows the main Wiki page for the RPi-Bot of the Web server monitoring system. This page includes the program for the RPi-Bot. The program do the followings

1. Tries to read the Web page of the Web server.

2. If the Bot could not read the page, tweet the following.

"rinri down <hour:min>"

3. If the Bot could read the page, tweet the following.

"rinri up <hour:min>"

\subsection{Server Room Monitoring System}

The server room monitoring system consists of a ADK-Bot, a RPi-Bot, 24 Wiki pages for for each hour in a day (H-WIKIs), 31 Wiki pages for each day in a month (D-WIKIs), and the class page. The ADK-Bot is placed in the server room for monitoring the environmental factors of the room. The ADK-Bot reads the $\mathrm{H}$-WIKI which is corresponsing to the current time, interprets the commands in the page, and writes back the current data of temperature and others to the H-WIKI. The class page is inserted into the D-WIKI of the current day. The class page contains the program which computes the average of data of every H-WIKI and writes back the results to the D-WIKI of the current day (Figure 9).

H-WIKIs and D-WIKIs have the link to a page which displays graphs of data in H-WIKIs and D-WIKIs. The manager can see the transitions of each item of data, such as the transition of temperature of the room. 


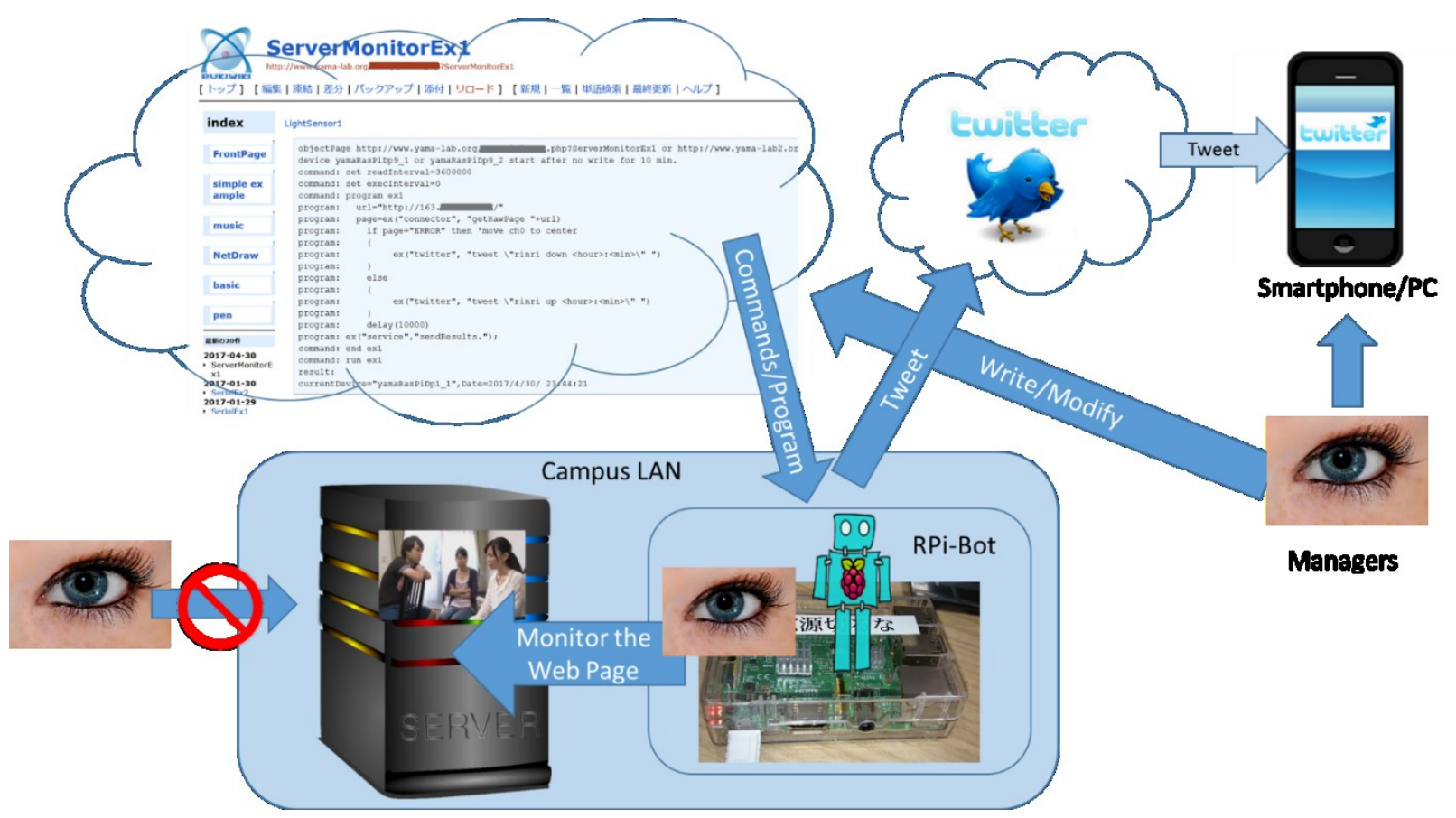

Figure 7: Web server monitoring system

\section{ServerMonitorEx1}

http://www.yama-lab.org ? ServerMonitorEx1

[トップ］［編集｜凍結｜差分 | バックアップ|添付 | リロード］［新規｜一覧｜単語検索 | 最終更新 | ヘルプ ]

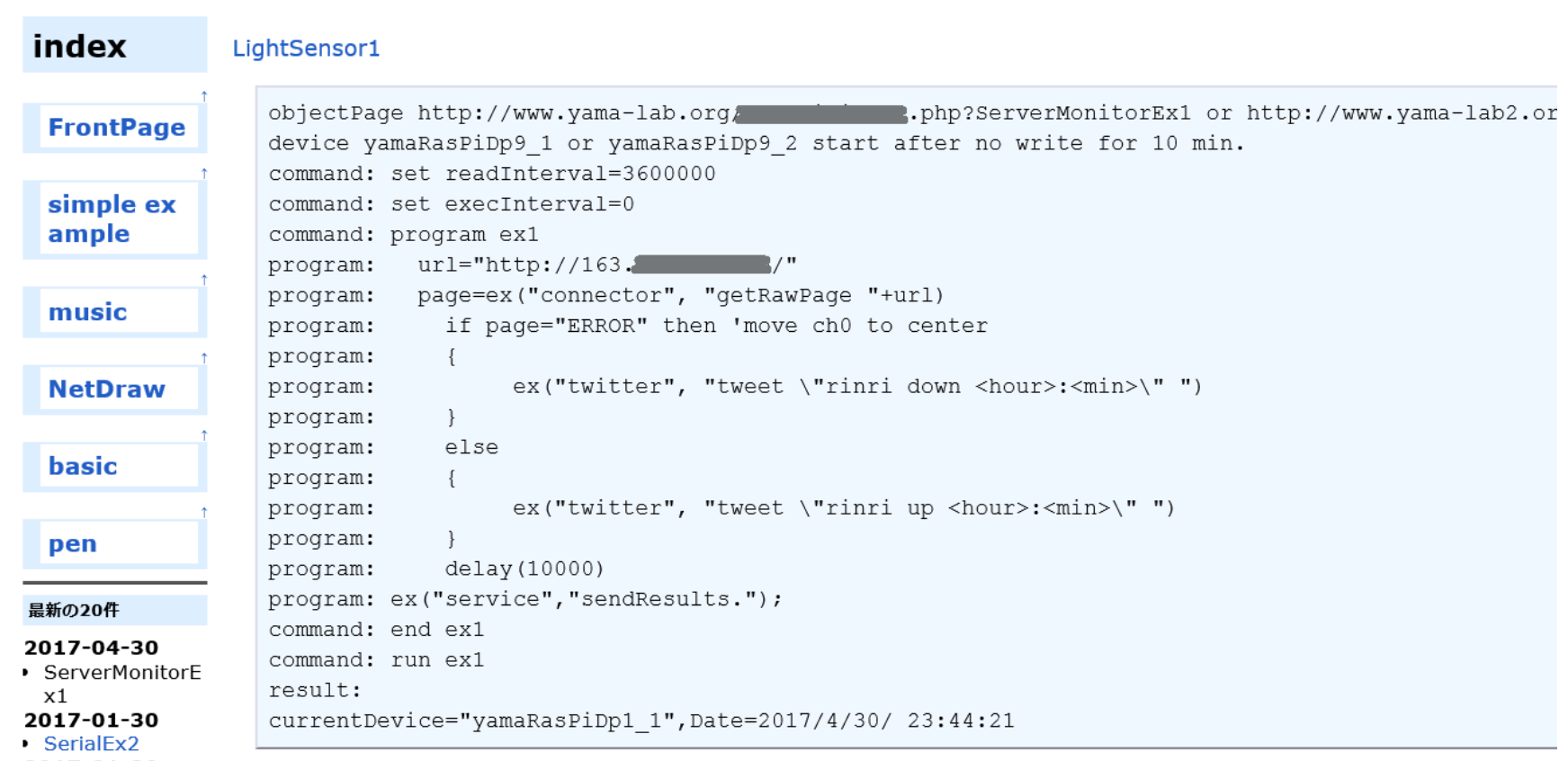

Figure 8: The program of the Web server monitoring system. 


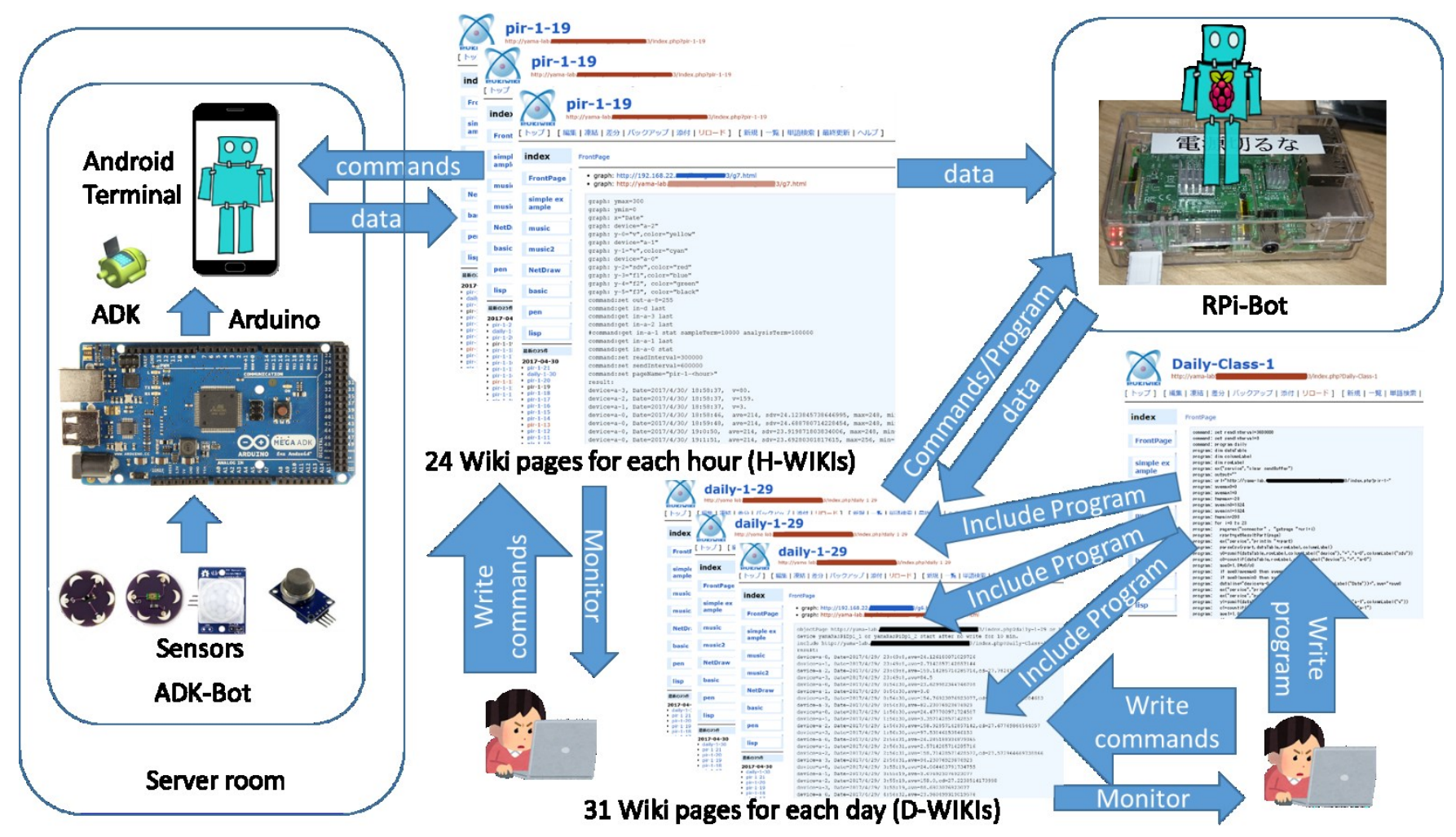

Figure 9: Server room monitoring system.

\section{HELP FROM MY BOTS}

We are using the Web server monitoring system for a half year and using the room server monitoring system for one and a half year. They are meeting our expectations.

Figure 10 shows the tweets which is tweeted by the RPi-Bot of the Web server monitoring system. The tweets alerted us that the Web server was down. We could see the Web server went down between 2:55 and 3:56 of March 18. It was a weekend and we were at home at that time. However, we went to the campus and fixed the problem.

Figure 11 and Figure 12 show the graphs of data in the server room. The graphs were generated by the server room monitoring system.

Figure 11 shows temperature, brightness, human motion and air pollution level in the server room during the day of April 27, 2017. The manager can see the graph by clicking the link to the graph page of the D-WIKI. On that date we can see that there was no temperature problem during the day. We believe someone entered the server room about noon because brightness of the room increased at that time. However, PIR-level, the human motion level, did not increase at the same time. This means that someone did not come close to the ADK-Bot.

Figure 12 shows the graph of the H-WIKI which is corresponding to the hour that seems someone enter the server room of the day of Figure 8. The manager can know the detail time of the changes by seeing the D-Wiki page and the graph.

Fortunately, there was no air conditioner trouble after the deployment of the server room monitoring system, so we could not test that aspect of the system.

\section{ALTERNATIVE MONITORING SOLUTIONS}

\subsection{Webalizer}

Webalizer[10] is a fast, free web server log file analysis program. It is popular tool for visualizing usage of a Web server. Webalizer is installed in the Web server which is the target of the visualization. So, the manager can't see visualized usage of the server from the outside of the network if the server is not accessible from the outside. On the other hand, our monitoring system provides managers the ability to monitor the target server from the outside. 


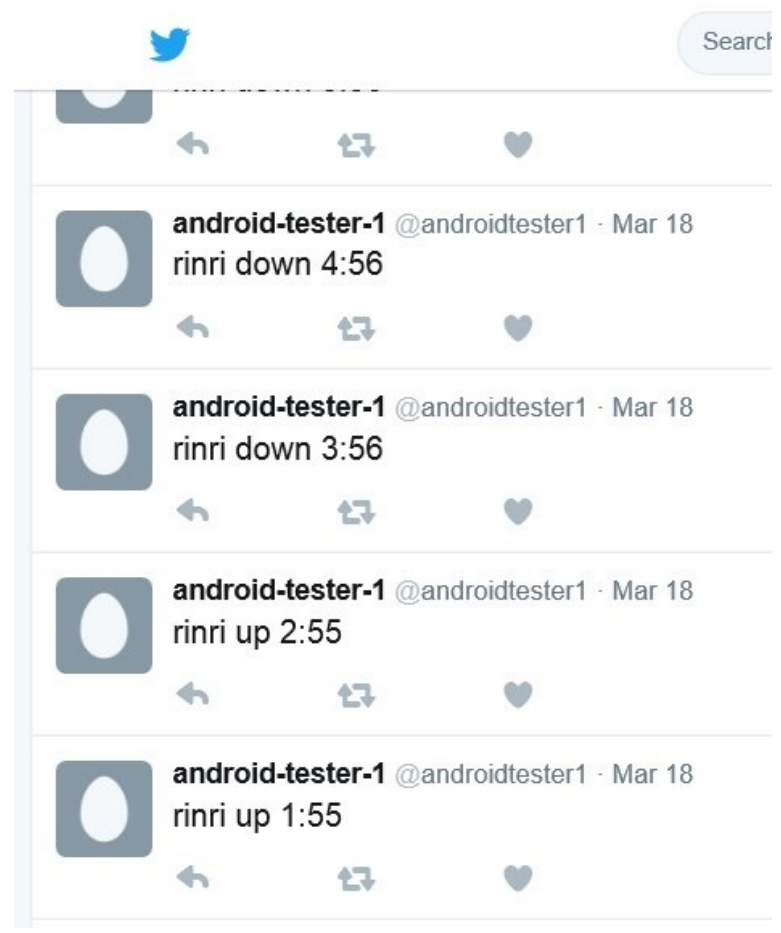

Figure 10: Tweets that show the Web server was down

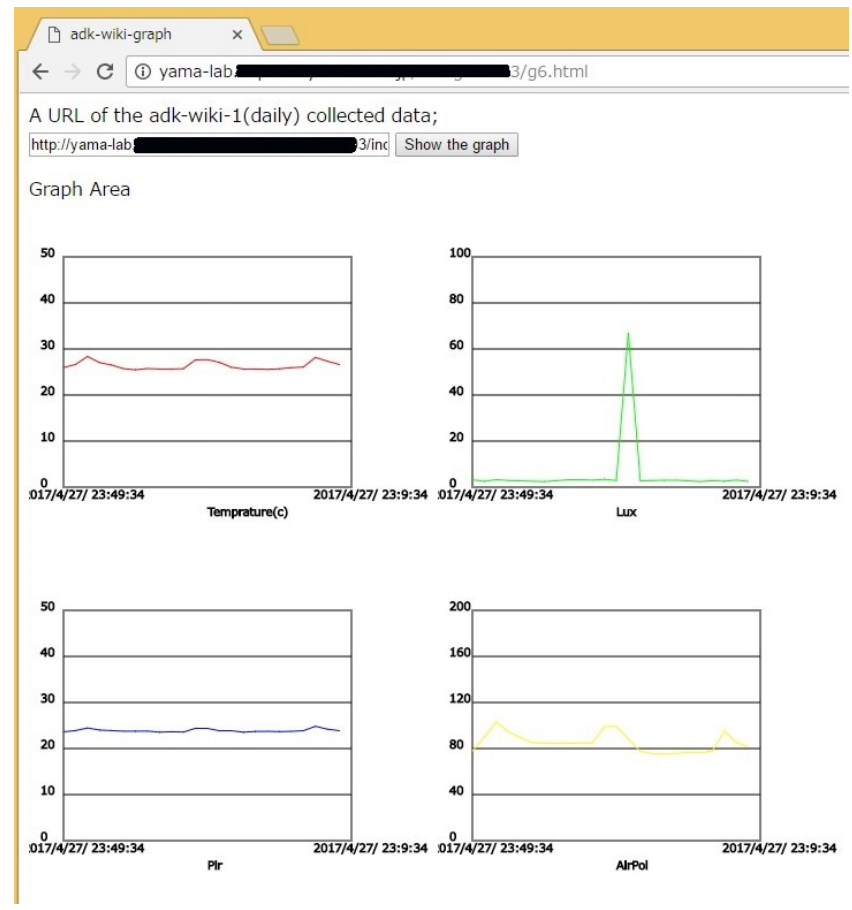

Figure 11: Transitions of temperature, brightness, human motion and air pollution level in the server room during the day of April 27, 2017.

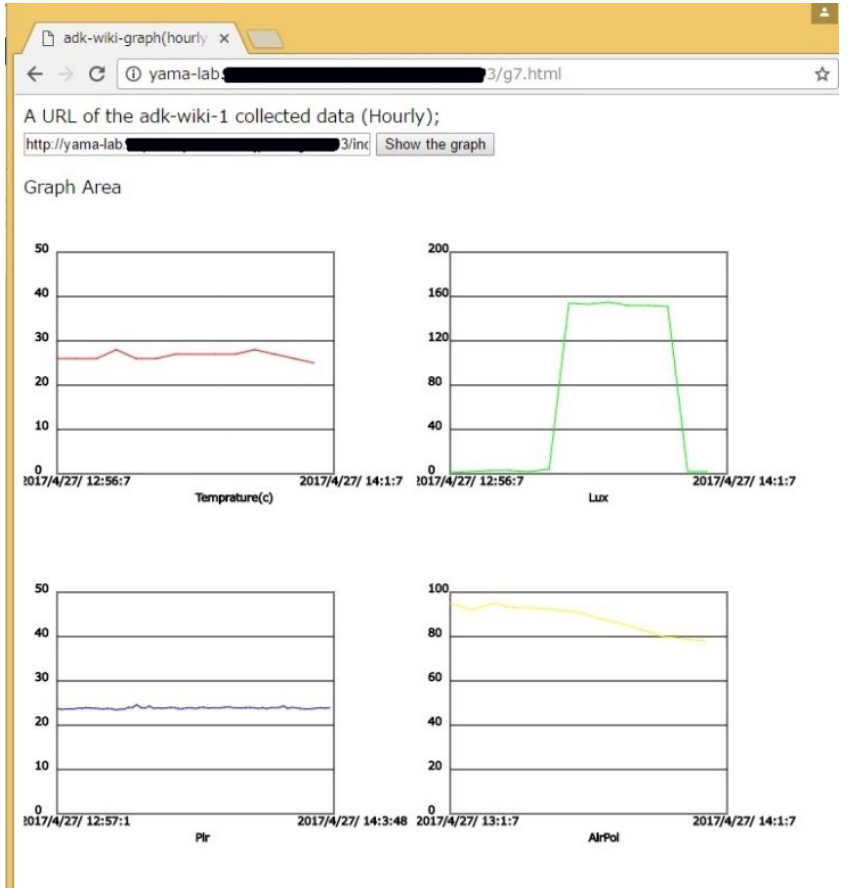

Figure 12: Transitions of temperature, brightness, human motion and air pollution level in the server room during

the hour which seems someone enter the server room.

\subsection{New Relic}

New Relic[11] is a popular commercial server monitoring service. New Relic can monitor almost all servers whether they are accessible from outside the network or not. The user of the New Relic can monitor the data at the New Relic's Web server. However, the monitoring program for New Relic has to be installed in the target server. On the other hand, our monitoring system allows managers to monitor the target server without installing a program on the target server.

\section{CONCLUSION}

We have shown our monitoring method, which uses a little help from our bots. We have also shown our results from using these bot monitoring systems.

Our bots helped to enhance the reliability of the target server and the target server room. Our bots also helped reduce our work load and mental stress.

There are several opportunities to improve our monitoring method, such as enhancing the security and enhancing the reliability. We would like to report our progress next time.

\section{ACKNOWLEDGMENTS}

A part of this research was supported by JSPS KAKENHI Grant Number JP16K00197. We also thank students who helped us to develop monitoring systems. 


\section{REFERENCES}

[1] Puri, R. 2003. Bots \& Botnet: An Overview," SANS InfoSec Reading Room, http://www.sans.org/rr/whitepapers/malicious/, Dec., 2003.

[2] Masuya, M., Yamanoue, T., Kubota, S. 2006. An Experience of Monitoring University Network Security Using a Commercial Service and DIY Monitoring. In Proceedings of the 34th Annual ACM SIGUCCS Conference on User Services (Edmonton, Alberta, Canada. 5-8 Nov. 2006). ACM, New York, NY, 225-230. DOI=http://doi.acm.org/10.1145/1181216.1181267

[3] Mattauch, T., Hatoum, R., Pettit, H. 2012. Building a call center in 2 days: how a world class support center responds to crisis. In Proceedings of the 40th Annual ACM SIGUCCS Conference on User Services (Memphis, Tennessee, USA. 15-19 Oct. 2012). ACM, New York, NY, 97-100 DOI=http://doi.acm.org/10.1145/2382456.2382478

[4] Yamanoue, T., Oda, K., Shimozono. K. 2012. Capturing Malicious Bots using a Beneficial Bot and Wiki, In Proceedings of the 40th annual ACM SIGUCCS conference on User services (Memphis, Tennessee, USA. 15-19 Oct. 2012). ACM, New York, NY, 91-96. DOI=https://doi.org/10.1145/2382456.2382477

[5] Takashi Yamanoue, Kentaro Oda, Koichi Shimozono. A Malicious Bot Capturing System using a Beneficial Bot and Wiki, Journal of Information Processing(JIP), vol.21, No.2, pp.237-245(2013)

[6] Yamanoue, T., Oda, K., Shimozono. K. 2013. An Inter-Wiki Page Data Processor for a M2M System, In Proceedings of the 4th International Conference on E-Service and Knowledge Management (ESKM 2013), Advanced Applied Informatics (IIAIAAI), 2013 IIAI International Conference on.(Matsue, Shimane, Japan, 31 Aug- 4 Sep. 2013) IEEE, Los Alamitos, CA. 45-50. DOI= https://doi.org/10.1109/IIAI-AAI.2013.48
[7] Yamanoue, T., Oda, K., Shimozono. K. 2014. Experimental Implementation of a M2M System Controlled by a Wiki Network, In Applied Computing and Information Technology,Studies in Computational Intelligence, Springer, Vol.553, 121-136.

[8] Yamanoue, T., Fuse, I., Okabe, S., Nakamura, A., Nakanishi, M., Fukada, S, Tagawa, T., Tatsumi, T., Murata, I, Uehara, T., Yamada, T., Ueda, H. 2014 Computer Ethics Video Clips for University Students in Japan from 2003 until 2013, In Proceedings of the 38th Annual International Computer Software \& Applications Conference (COMPSAC2013/ADMNET WS) (Västerås, Sweden, 21-25 Jul. 2014). IEEE, NJ, 96-101.

[9] Yamanoue, T., Nakamichi, N., Kaneko, K., 2017. Enhancing Campus Cyber Security through a Class with Combination of Computer Ethics Videos and Logical Thinking. In SIGUCCS'16 Proceedings of the ACM on SIGUCCS Annual Conference (Denver, Colorado, USA, 6-9 Nov. 2016). ACM, New York, NY. 117-123. DOI=http://doi.acm.org/10.1145/2974927.2974939

[10] Webalizer, http://www.webalizer.org, as of 4 May, 2017

[11] New Relic,https://newrelic.com/, as of 4 May, 2017 